\title{
Noninvasive measurement of cerebrospinal fluid flow using an ultrasonic transit time flow sensor: a preliminary study
}

\author{
Thomas Pennell, MS, ${ }^{1}$ Juneyoung L. Yi, MD, ${ }^{2}$ Bruce A. Kaufman, MD, ${ }^{3}$ and \\ Satish Krishnamurthy, MD, MCh ${ }^{2}$
}

${ }^{1}$ Transonic Systems Inc., Ithaca; and 2Department of Neurosurgery, SUNY Upstate Medical University, Syracuse, New York; and ${ }^{3}$ Department of Neurosurgery, Medical College of Wisconsin, Milwaukee, Wisconsin

\begin{abstract}
OBJECTIVE Mechanical failure-which is the primary cause of CSF shunt malfunction-is not readily diagnosed, and the specific reasons for mechanical failure are not easily discerned. Prior attempts to measure CSF flow noninvasively have lacked the ability to either quantitatively or qualitatively obtain data. To address these needs, this preliminary study evaluates an ultrasonic transit time flow sensor in pediatric and adult patients with external ventricular drains (EVDs). One goal was to confirm the stated accuracy of the sensor in a clinical setting. A second goal was to observe the sensor's capability to record real-time continuous CSF flow. The final goal was to observe recordings during instances of flow blockage or lack of flow in order to determine the sensor's ability to identify these changes.
\end{abstract}

METHODS A total of 5 pediatric and 11 adult patients who had received EVDs for the treatment of hydrocephalus were studied in a hospital setting. The primary EVD was connected to a secondary study EVD that contained a fluid-filled pressure transducer and an in-line transit time flow sensor. Comparisons were made between the weight of the drainage bag and the flow measured via the sensor in order to confirm its accuracy. Data from the pressure transducer and the flow sensor were recorded continuously at $100 \mathrm{~Hz}$ for a period of 24 hours by a data acquisition system, while the hourly CSF flow into the drip chamber was recorded manually. Changes in the patient's neurological status and their time points were noted.

RESULTS The flow sensor demonstrated a proven accuracy of $\pm 15 \%$ or $\pm 2 \mathrm{ml} / \mathrm{hr}$. The flow sensor allowed real-time continuous flow waveform data recordings. Dynamic analysis of CSF flow waveforms allowed the calculation of the pressure-volume index. Lastly, the sensor was able to diagnose a blocked catheter and distinguish between the blockage and lack of flow.

CONCLUSIONS The Transonic flow sensor accurately measures CSF output within $\pm 15 \%$ or $\pm 2 \mathrm{ml} / \mathrm{hr}$, diagnoses the blockage or lack of flow, and records real-time continuous flow data in patients with EVDs. Calculations of a wide variety of diagnostic parameters can be made from the waveform recordings, including resistance and compliance of the ventricular catheters and the compliance of the brain. The sensor's clinical applications may be of particular importance to the noninvasive diagnosis of shunt malfunctions with the development of an implantable device.

Clinical trial registration no.: NCT00652197 (clinicaltrials.gov)

http://thejns.org/doi/abs/10.3171/2015.7.PEDS1577

KEY WORDS hydrocephalus; shunt malfunction; ultrasonic transit time flow sensor; pressure-volume index

$\mathrm{M}$ ORE than 125,000 US patients are diagnosed with hydrocephalus (HCP) - a condition that demands lifelong treatment, often with implanted shunt systems to drain excess CSF. ${ }^{15}$ Despite the many advances in modern neurosurgery, shunts continue to malfunction. This holds true particularly in children, as this demographic has a $25 \%$ to $40 \%$ chance of shunt failure within the 1 st year of implantation. ${ }^{2,17}$ The most common occurrence in the general shunted population is mechanical failure, including obstruction, disconnection of the catheters and/or valves, valve malfunction/occlusion, underdrainage, and overdrainage. The rate of mechanical malfunction ranges from $8 \%$ to $64 \% .{ }^{19}$

Due to such high rates, shunts continue to comprise a

ABBREVIATIONS EVD = external ventricular drain; HCP = hydrocephalus; ICP = intracranial pressure; PVI = pressure-volume index; SUNY = State University of New York.

SUBMITTED February 4, 2015. ACCEPTED July 13, 2015.

INCLUDE WHEN CITING Published online November 13, 2015; DOI: 10.3171/2015.7.PEDS1577. 
large part of medical care in the United States. Annually, there are roughly 39,000 admissions that account for as many as 433,000 hospital days for pediatric HCP alone. The annual burden for HCP-related shunts ranges from $\$ 1.4$ to $\$ 2$ billion per year, and nearly half of these expenses go toward the revision of malfunctioning systems. ${ }^{15}$

Unfortunately, there is still no means to obviate this burden - a single, noninvasive but accurate device for detecting mechanical malfunction does not exist. Instead, the clinician is often forced to perform several tests, including MRI, CT, shunt flow studies ("shunt-o-grams"), and shunt taps. These tests increase radiation exposure to patients ${ }^{6}$ and potentially introduce infection into shunt systems, resulting in surgical removal and thus prolonging hospital courses and further increasing costs..$^{13}$ Furthermore, the specific reason for mechanical failure-absent, excessive, or inadequate CSF flow-often eludes the clinician. ${ }^{19}$

This study evaluates the use of an external, ultrasonic, transit time flow sensor to address this unmet need. The initial implantable version of this had been developed (Transonic Systems Inc.), which connects in-line to the existing shunt tubing and can measure flow transcutaneously via inductive signal coupling. The flow sensor has a resolution of $0.5 \mathrm{ml} / \mathrm{hr}$ and accuracy greater than $\pm 15 \%$ or $\pm 2 \mathrm{ml} / \mathrm{hr}$ (after zero offset adjustment) in bench testing. To validate the flow measurement technology in an external, nonimplant setting, Transonic Systems developed a similar transit time flow sensor that is connected in-line to an EVD system and does not require inductive signal coupling to record measurements.

This paper documents the results of an observational clinical study conducted on 5 pediatric patients at Children's Hospital of Wisconsin and 11 adult patients at the State University of New York (SUNY) Upstate Medical University using this EVD flow sensor. Two specific cases at Children's Hospital of Wisconsin illustrate the potential value of flow measurement in HCP patients with implanted shunts (clinical trial registration no.: NCT00652197; http://www.clinicaltrials.gov).

\section{Methods \\ Patients}

A total of 5 pediatric and 11 adult patients who were being treated for HCP were studied. Patients were included if they could be connected to a single, patent EVD at the time of study start. Table 1 lists the patient demographics, including age, sex, and reason for EVD insertion. The clinical team was not blind to this information.

\section{Study Protocol}

The study protocol was approved by the institutional review boards at both the Children's Hospital of Wisconsin and SUNY Upstate Medical University. The protocol followed adheres to principles set forth in the US Code of Federal Regulations, Title 45, Part 46, Protection of $\mathrm{Hu}$ man Subjects, revised January 15, 2009 (FWA 00002071, FWA 00005967). Informed consent was obtained from each enrolled patient. Consent was obtained either from the patient or the patient's health care proxy in the cases of underage and incapacitated individuals.
TABLE 1. Patient demographics

\begin{tabular}{cccl}
\hline Case No. & Age (yrs) & Sex & \multicolumn{1}{c}{ Reason for EVD } \\
\hline Peds cases & & & \\
\hline 1 & 17 & M & Poor abdominal CSF absorption \\
\hline 2 & 10 & M & Shunt infection \\
\hline 3 & 16 & F & Shunt infection \\
\hline 4 & 11 & M & Shunt infection \\
\hline 5 & 14 & F & Postoperative edema after Chiari \\
& & & decompression \\
\hline Adult cases & & & \\
\hline 1 & 53 & M & Subarachnoid hemorrhage \\
\hline 2 & 41 & M & Aneurysmal subarachnoid hemorrhage \\
\hline 3 & 49 & F & Aneurysmal subarachnoid hemorrhage \\
\hline 4 & 65 & M & Anoxic brain injury \\
\hline 5 & 72 & F & Aneurysmal subarachnoid hemorrhage \\
\hline 6 & 64 & M & Aneurysmal subarachnoid hemorrhage \\
\hline 7 & 71 & F & Aneurysmal subarachnoid hemorrhage \\
\hline 8 & 48 & F & Aneurysmal subarachnoid hemorrhage \\
\hline 9 & 78 & F & Intraparenchymal hemorrhage \\
\hline 10 & 75 & F & Intraparenchymal and intraventricular \\
& & & hemorrhage \\
\hline 11 & 54 & F & Aneurysmal subarachnoid hemorrhage \\
\hline
\end{tabular}

Peds $=$ pediatric

* Pediatric patients were enrolled at Children's Hospital of Wisconsin.

† Adult patients were enrolled at SUNY Upstate Medical University.

Patients already connected to an EVD as a treatment for HCP were connected to a secondary study EVD via a stopcock connection (Fig. 1). The secondary EVD contained a fluid-filled pressure transducer (TruWave, Edwards Lifesciences) and an in-line transit time flow sensor (Transonic Systems Inc.). A secondary EVD system was used to maintain the sterility of the primary EVD system and allow rapid disconnection of the study EVD in the event the patient needed to be moved for imaging or a procedure. In these cases, the study would be terminated, and the patient would not be reconnected to the study EVD system. The pediatric patients at Children's Hospital of Wisconsin were connected to a Medtronic Becker EDMS II EVD system, while adult patients at SUNY Upstate were connected to an Integra DUET EVD system for both the primary and secondary EVDs.

The drainage bag on the study EVD was weighed prior to the EVD being connected at the beginning of the study and again at the end of the study after disconnection from the primary EVD in order to allow weight-based comparisons with the flow measured by the transit time flow sensor. The study EVD was primed with sterile $0.9 \%$ saline and connected to the primary EVD via a stopcock connection located at the drip chamber of the primary EVD. The transit time flow sensor was connected to a custom Transonic flowmeter (TS420), and the fluid-filled Transpak IV pressure transducer (Hospira) was connected to a Transonic pressure monitor (TS430). Data from the pressure transducer was collected continuously on an open drain. The reason for recording the intracranial pres- 


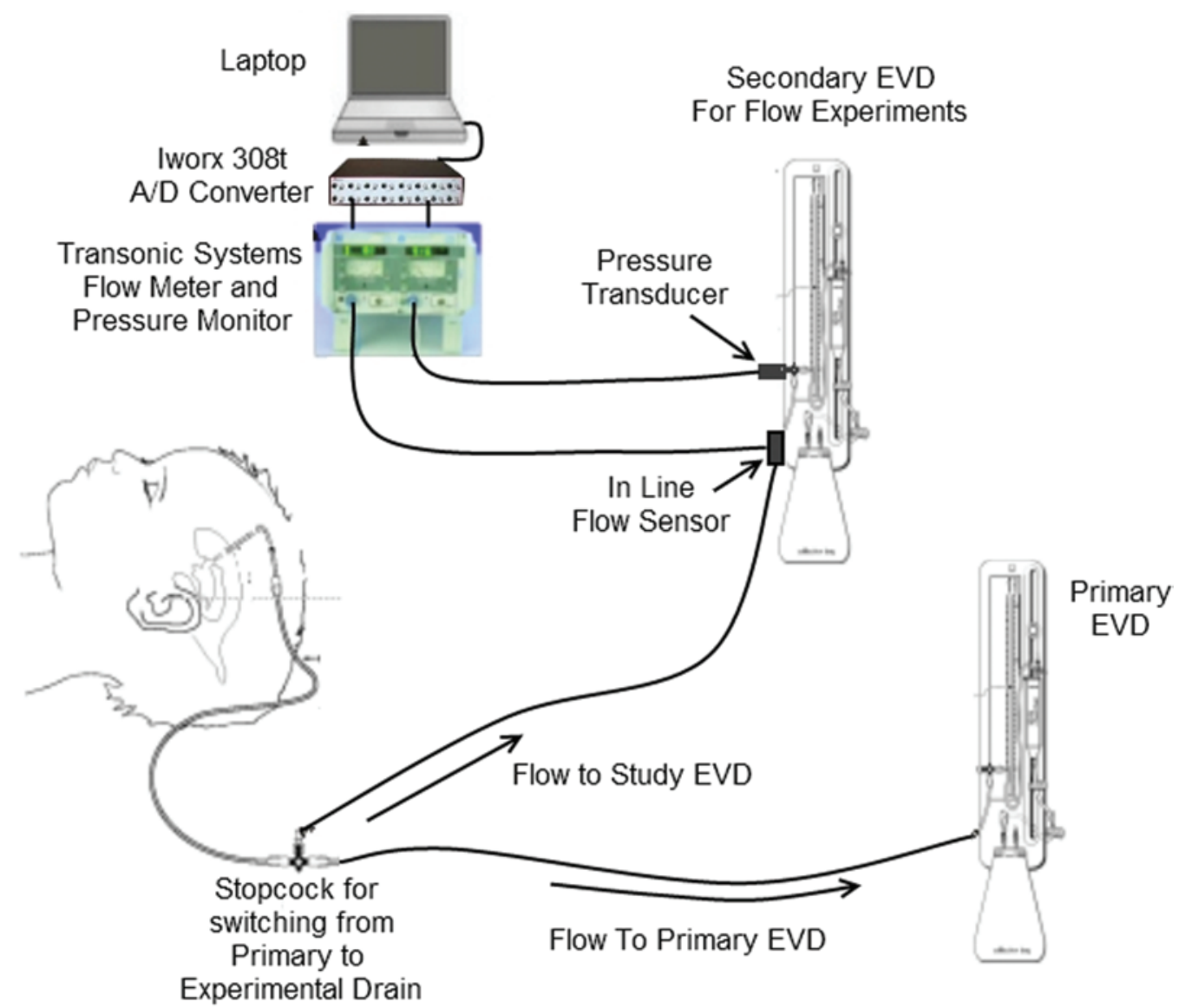

FIG. 1. Study setup. Figure is available in color online only.

sure (ICP) on the open drain was to combine flow measurement with the known resistance of the shunt tubing in order to apply an established circuit model to the data; this allowed the calculation of other diagnostic parameters. The flow sensor and pressure transducer were properly zeroed, and flow was diverted to the study EVD via the stopcock. Flow, pressure, temperature, and other signals that serve as flow sensor diagnostics were recorded continuously at $100 \mathrm{~Hz}$ for a period of 24 hours for each patient. Data were recorded using a 308T data acquisition system (Iworx) that was connected to the Transonic flow and pressure meters. CSF flow to the drip chamber was also recorded manually by the nursing staff during hourly patient evaluations.

The clinical team was not blind to the ICP measurements and CSF volume output. However, clinical investigators were blind to the flow data, and nonclinical investigators carried out subsequent analyses of all parameters after data collection had been terminated.

\section{Results}

Data comparing weight-based CSF flow and the flow calculated by the flow sensor at each clinical site are shown in Table 2, respectively. The data show that the flow sensor had an accuracy of $\pm 15 \%$ or $\pm 2 \mathrm{ml} / \mathrm{hr}$ (after zero offset adjustment). One patient was excluded from the data set at Children's Hospital of Wisconsin due to a measurement interruption during the study period. Two patients were excluded from the SUNY Upstate Medical Center data set due to interruption of the study, and 1 patient was excluded due to a nursing error during the study.

Fourier analysis was conducted on the flow and pressure waveforms in each patient. Such an analysis transforms the periodic signaling found in a wave into the sum of the waves and thus a linear composition. The data can then be represented in terms of frequency instead of time. The purpose of the Fourier analysis in this study was to identify the most commonly occurring frequencies in the acquired waveforms and to then relate these frequencies to physiological parameters. The analysis coincided with the hourly measurements obtained by the nursing staff of the heart rate and respiratory rate; thus, the pulsatility of the acquired data was proven to be comparable with these physiological parameters.

Data shown in Fig. 2A and B were taken from a 41-yearold, 110.3-kg male patient who was admitted to SUNY Upstate Medical University. These data illustrate the typical CSF flow and ICP waveforms observed during the study. Flow measurement was started 10 days after admission, and the patient was considered to be in stable condition at the time. Fourier analysis was conducted on the flow and pressure waveforms shown in Fig. 2A and B in order to compare the values for respiration and heart rate obtained 
TABLE 2. Comparison of CSF collected during a 24-hour period by weight versus volume, as calculated by the in-line flow sensor prototype

\begin{tabular}{cccc}
\hline Case No. & $\begin{array}{c}\text { Fluid Weight } \\
\text { by Scale }(\mathrm{g})\end{array}$ & $\begin{array}{c}\text { Fluid Measured } \\
\text { by Sensor }(\mathrm{ml})\end{array}$ & \% Error \\
\hline Peds cases* & & & \\
\hline 1 & 233.7 & 213.4 & -8.6 \\
\hline 2 & 178.3 & 166.7 & -6.5 \\
\hline 4 & 467.3 & 417.6 & -10.6 \\
\hline 5 & 141.8 & 138.8 & -2.1 \\
\hline Mean & 255.3 & 234.12 & $-6.95 \pm 3.64$ \\
\hline Adult cases $\dagger$ & & & \\
\hline 1 & 301.7 & 269.1 & -10.8 \\
\hline 2 & 261.4 & 230.1 & -11.95 \\
\hline 3 & 265.9 & 276.1 & 3.8 \\
\hline 7 & 119 & 112.2 & -5.69 \\
\hline 8 & 71.5 & 73.9 & 3.31 \\
\hline 9 & 121.7 & 119.2 & -2.06 \\
\hline 10 & 141.2 & 142.2 & 0.7 \\
\hline 11 & 329.6 & 318.5 & -3.3 \\
\hline Mean & 201.5 & 192.7 & $-3.25 \pm 5.96$ \\
\hline * Pediatric Case 3 was excluded due to a measurement interruption during \\
the study. \\
$\begin{array}{l}\text { Adult Cases 4 and 5 were excluded due to interruptions during the study, } \\
\text { and adult Case 6 was excluded due to a nursing error. }\end{array}$ \\
\hline
\end{tabular}
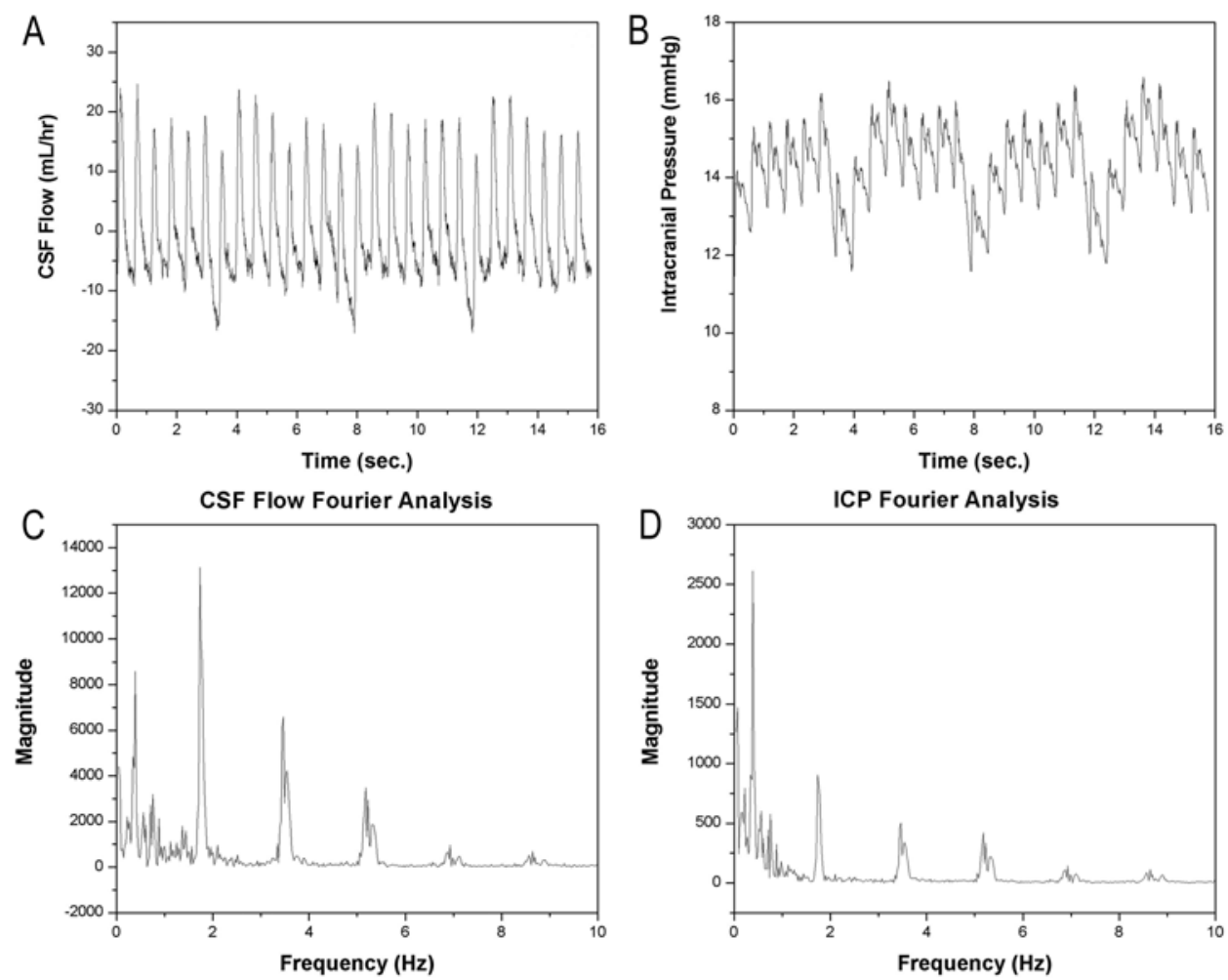

FIG. 2. Continuous monitoring in a 41-year-old male patient admitted for aneurysmal subarachnoid hemorrhage. A: Typical pulsatile CSF flow waveform. B: ICP waveform. C: Fourier analysis of CSF flow. D: Fourier analysis of the ICP waveform. 
This case illustrates the flow sensor's ability to differentiate between impaired CSF circulation and absorption, leading to high flow through the EVD with high-amplitude pulsatility, and normal CSF circulation following drainage, which manifests as low flow through the shunt with a diminished pulse amplitude.

\section{Case 2}

A 17-year-old, 62.3-kg male patient was admitted to Children's Hospital of Wisconsin for ventriculoperitoneal shunt malfunction due to poor abdominal absorption. The shunt was removed, and the patient received an EVD while awaiting the implantation of a new shunt system. The patient was subsequently connected to the study EVD.

The patient complained of a headache at approximately 4.75 hours after the start of the study. Physical examination revealed that the shunt catheter had become kinked at its exit point. The catheter was unkinked and then sutured to the scalp to prevent rekinking. Figure $4 \mathrm{~A}-\mathrm{G}$ illustrates the flow sensor's ability to not only detect a shunt blockage but also detect blockage prior to the patient becoming symptomatic.

\section{Discussion}

Implanted CSF shunts are plagued with problems and associated with a high rate of failure. Catheter blockage, infection, and valve malfunction are all contributors to shunt malfunction. Shunt failure in pediatric cases has been reported to be $31 \%$ within the first year and $4.5 \%$ per year thereafter. ${ }^{16}$ This poses a significant problem, as the differential diagnosis of shunt malfunction warrants a battery of tests, each with its own limitations. This leads to increased treatment costs, prolonged hospital stays, increased radiation exposure, and additional surgical courses that are often unnecessary. ${ }^{5,6,14}$ Thus, the need for a noninvasive but accurate tool to diagnose mechanical malfunction remains.

Prior attempts to noninvasively measure CSF flow have been made. A study that performed the weight-based measurement of the EVD system, akin to this study, was limited in that the frequency of data acquisition $(1 \mathrm{~Hz})$ did not allow analysis of the pulsatile nature of the flow waveform. ${ }^{3}$ Radionuclide injections into an implanted shunt reservoir were able to detect shunt patency. However, measurements could only be repeated a finite number of times and were not quantitative. This method also did not analyze the pulsatile nature of CSF flow dynamics and exposed the patient to repeated doses of radiation. ${ }^{1,4,11,18}$ MRI techniques allow the study of the pulsatile nature of CSF flow, but they provide data during a single cardiac cycle as opposed to continuous monitoring. Furthermore, MRI continues to be an expensive and time-consuming study that is not well tolerated by many patients due to claustrophobia. Most importantly, many shunt valves contain magnetic components that have the potential to alter valve settings and cause artifacts on acquired images. ${ }^{10,12}$ The ShuntCheck device (NeuroDx Development) uses a thermal technique to noninvasively evaluate flow through an implanted shunt. While the technique has an $80 \%$ specificity for flow, the "detection of flow does not statistically
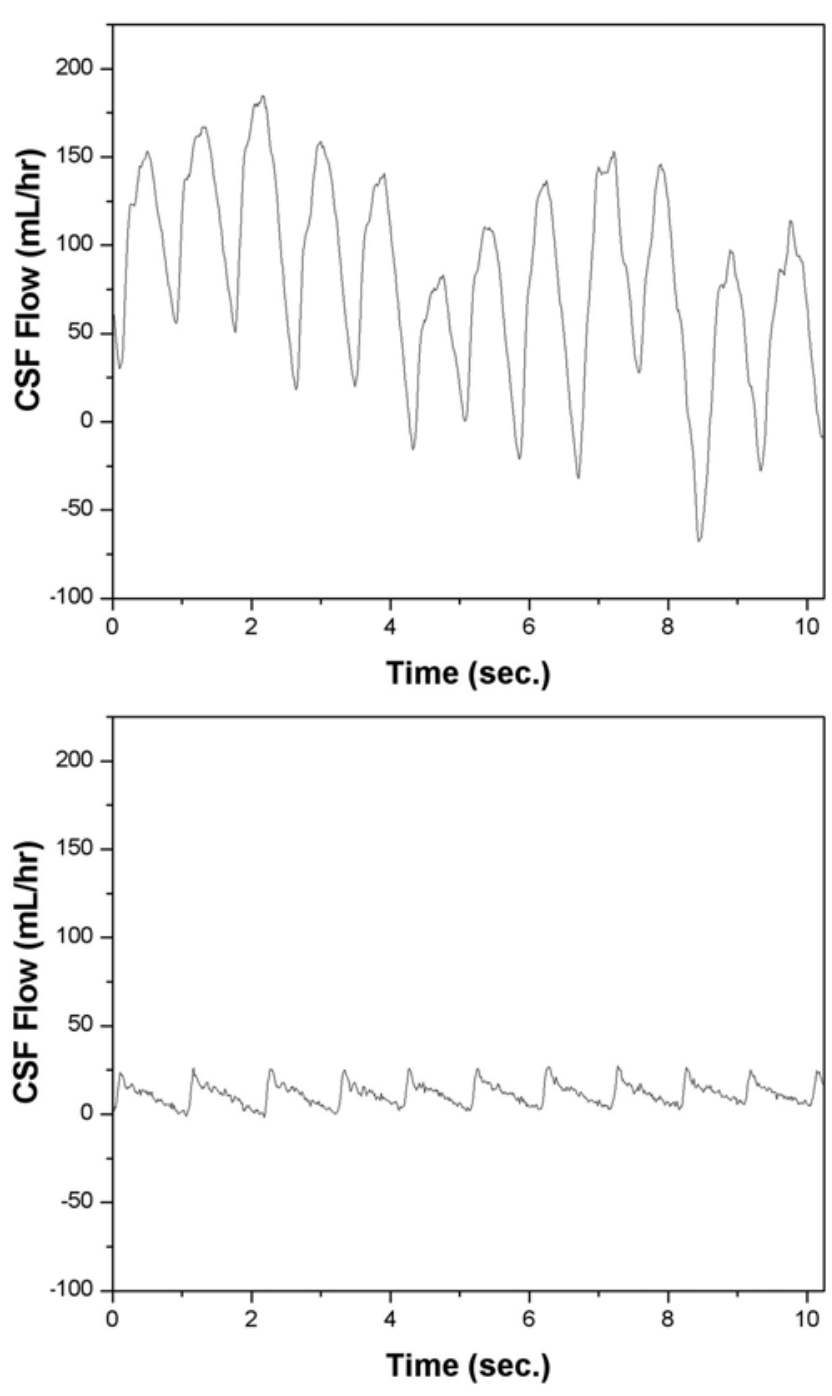

FIG. 3. CSF flow measured pre- and postrecovery in a pediatric patient who experienced postoperative swelling after suboccipital decompression for Chiari malformation.

predict clinical diagnosis of shunt failure." Also, "further understanding of the quantitative aspects of flow could be acquired under those circumstances where an alternative method of quantitatively visualizing flow is possible."

This study confirms that the Transonic sensor is capable of automatic, real-time measurements of flow and CSF drainage volume through an EVD with an accuracy $\pm 15 \%$ or $\pm 2 \mathrm{ml} / \mathrm{hr}$ over long periods of time. Thus, it is not reliant on hospital staff and can document these parameters at any time point. This is contrasted with the pressure transducer, which requires the manual closure of the drainage system in order to allow hourly recordings over just a few minutes. The data obtained can be used to calculate multiple other useful clinical parameters, including PVI, compliance, the resistance of the EVD catheter, and even brain compliance. Lastly, the specific cases described above demonstrate the sensor's ability to diagnose a blocked catheter. More importantly, the sensor can differentiate between blockage and lack of flow. In the case of 

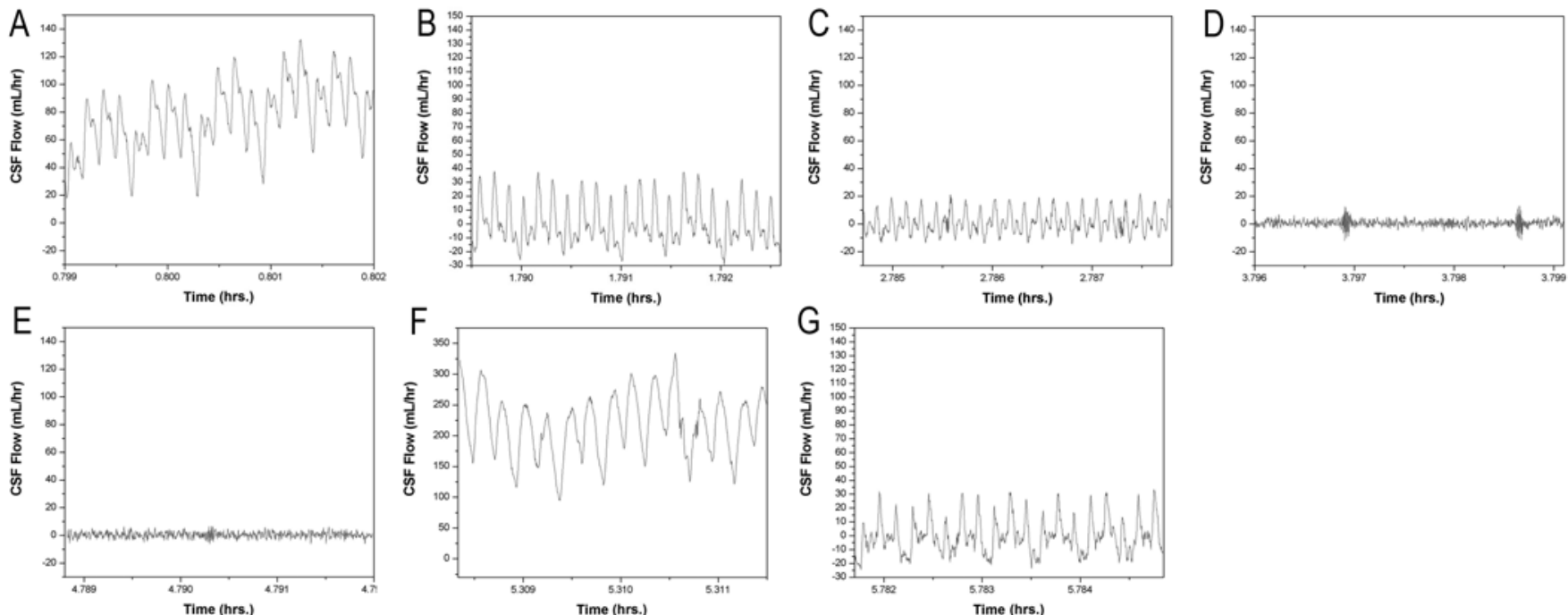

FIG. 4. CSF flow waveforms in a 17-year-old patient who experienced CSF flow blockage secondary to the kinking of the EVD. A: Upon first opening the EVD at the time the study was started (high flow and amplitude of pulsatility). B: After approximately 1.75 hours of continuous flow (normal mean flow and amplitude of pulsatility). C: After approximately 2.75 hours of continuous flow (diminished mean flow and amplitude of pulsatility). D: Complete blockage of the proximal catheter at approximately 3.75 hours (loss of waveform). E: Continued blockage of the proximal catheter at approximately 4.75 hours. F: Upon unkinking the proximal catheter at 5.3 hours (restoration of waveform with high flow and amplitude of pulsatility). G: Reestablishment of unimpeded flow through the catheter at approximately 5.75 hours (normalization of waveform).

the former, the pulsatile flow waveform would be absent; in the case of the latter, the waveform would be present but with diminished mean flow and amplitude.

Dynamic analysis of CSF flow waveforms may elucidate other important diagnostic parameters. These include
PVI, intracranial compliance, and ICP, thus giving physicians further noninvasive diagnostic ability.

The circuit model shown in Fig. 5 is a modified version of the Marmarou model published in 1978. ${ }^{8,9}$ Additions to this model have been made to include the components of

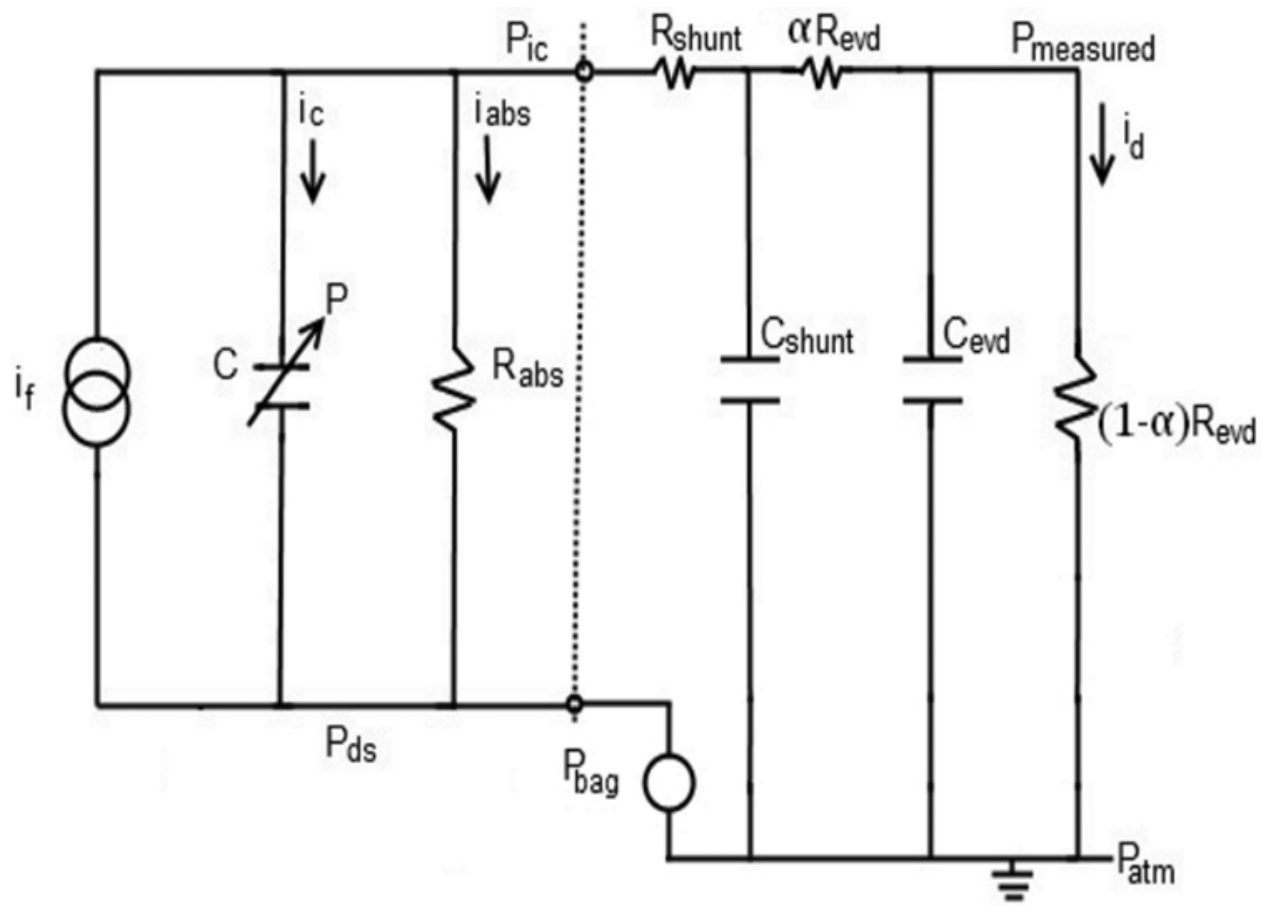

FIG. 5. Circuit model of the CSF system modified to include drainage to the EVD. See Discussion for the definitions of the terms used in this figure. 
the EVD system. Similar modifications to the model can be made to include the components of an implanted shunt.

In this model, the formation of CSF is represented by a constant flow generator $\left(\mathrm{q}_{\mathrm{f}}\right)$. CSF storage is represented by nonlinear capacitance (C) and has an associated storage flow into the distending tissue $\left(i_{c}\right)$. Reabsorption of CSF is represented by resistance $\left(\mathrm{R}_{\mathrm{abs}}\right)$, facilitating an associated reabsorption flow $\left(i_{\text {abs }}\right)$. Lastly, ICP and dural sinus pressure are represented by $P_{\text {ic }}$ and $P_{d s}$, respectively. The circuit diagram is sketched with its equivalent symbols: current is analogous to flow, pressure to voltage, and capacitance to the compliance of the brain compartment. Circuit components are defined in equivalent fashion from pressure-flow relationships, such as $\mathrm{p}=\mathrm{q} \times \mathrm{R}$ and $\mathrm{q}=\mathrm{C} \times(\mathrm{dP} / \mathrm{dt})$.

These terms also apply to the EVD system, where the ventricular catheter and EVD tubing have resistance and compliance ( $\mathrm{R}_{\text {shunt }}, \mathrm{C}_{\text {shunt }}, \mathrm{R}_{\mathrm{EVD}}$, and $\mathrm{C}_{\mathrm{EVD}}$, respectively). There are several pressures in the EVD system that need to be taken into account, including atmospheric pressure $\left(\mathrm{P}_{\text {atm }}\right)$ and the hydrostatic pressure associated with the height setting of the EVD system $\left(\Delta \mathrm{P}_{\text {bag }}\right)$.

Using this circuit model combined with flow measurement $\left(i_{d}\right)$, it is now possible to calculate a wide variety of diagnostic parameters. For example, by knowing the resistance and compliance of the ventricular catheter and EVD tubing, it is possible to calculate the actual intracranial pressure with the EVD line open using the following equation: $i_{d}=\left(P_{\text {ic }}-P_{\text {measured }}\right) /\left(R_{\text {shunt }}+R_{E V D}\right)$. The rate of reabsorption of CSF by natural means can then be calculated knowing the dural sinus pressure $\left(\mathrm{P}_{\mathrm{ds}}\right)$ using the following equation: $i_{a b s}=\left(P_{i c}-P_{d s}\right) / R_{a b s}$. It will also be possible to calculate the compliance of the brain connected to an EVD in a noninvasive manner.

In addition, whereas prior PVI measurements described in the literature ${ }^{14,16}$ were potentially dangerous to the patient, thereby requiring a bolus infusion of fluid into the ventricles of a patient with an already elevated ICP, this measurement could be done using a minimally invasive approach. By allowing the CSF to drain while integrating the flow signal, one can measure a known amount of fluid leaving the ventricles. By combining this measurement with pressure measurement, both before and after passive drainage, one can calculate PVI without putting the patient at risk for further elevated ICP or infection.

The study was limited in power and by its purely observational design. Data would need to be collected in a larger number of patients and for periods of time longer than 24 hours. Furthermore, the flow sensor was assessed in patients of varying etiologies for HCP. Rather, accuracy would have to be established in each subset of HCP patients and then in comparison studies among these subsets.

Further studies would need to include data obtained from changes in EVD height using a similar external flow sensor-EVD setup. Continuous monitoring would also have to be performed in the setting of programmable valves. Two of the more important areas of additional investigation include recordings from implanted flow sensor devices and comparison studies with the current gold standard for temporary CSF diversion technology-pressure transducers.

\section{Conclusions}

The Transonic flow sensor was proven to accurately measure CSF flow, diagnose blockage versus lack of flow, and record real-time continuous flow data in patients with EVDs, thus providing physicians the ability to calculate a host of new diagnostic parameters. An implantable flow sensor placed in-line with a shunt system might be a method for noninvasively detecting shunt malfunctions. If this method were successfully implemented, it could decrease the number of diagnostic tests performed, obviate the need for potentially harmful diagnostic techniques, and decrease the length of hospital stay.

\section{Acknowledgments}

The hardware development and clinical validation studies of this project were funded by Phase I and Phase II Small Business Innovation Research (SBIR) grants of the National Institutes of Health: National Institute of Neurological Disorders and Stroke awarded to Transonic Systems Inc. ("A Flow Monitor for Pediatric Hydrocephalus Shunts"-\#2R44NS049680-02 and \#5R44NS049680-03).

We would like to acknowledge the nursing staff at Children's Hospital of Wisconsin and SUNY Upstate Medical University for their assistance in patient care and data collection.

\section{References}

1. Chervu S, Chervu LR, Vallabhajosyula B, Milstein DM, Shapiro KM, Shulman K, et al: Quantitative evaluation of cerebrospinal fluid shunt flow. J Nucl Med 25:91-95, 1984

2. Drake JM, Sainte-Rose C: The Shunt Book, ed 1., Cambridge, MA: Blackwell Science, 1995

3. Drake JM, Sainte-Rose C, DaSilva M, Hirsch JF: Cerebrospinal fluid flow dynamics in children with external ventricular drains. Neurosurgery 28:242-250, 1991

4. Elisevich K, Mattar AG, Cheeseman F: Biodegradation of distal shunt catheters. Pediatr Neurosurg 21:71-76, 1994

5. Hall EJ: Lessons we have learned from our children: cancer risks from diagnostic radiology. Pediatr Radiol 32:700-706, 2002

6. Krishnamurthy S, Schmidt B, Tichenor MD: Radiation risk due to shunted hydrocephalus and the role of MR imagingsafe programmable valves. AJNR Am J Neuroradiol 34:695-697, 2013

7. Madsen JR, Abazi GS, Fleming L, Proctor M, Grondin R, Magge S, et al: Evaluation of the ShuntCheck noninvasive thermal technique for shunt flow detection in hydrocephalic patients. Neurosurgery 68:198-205, 2011

8. Marmarou A, Shulman K, LaMorgese J: Compartmental analysis of compliance and outflow resistance of the cerebrospinal fluid system. J Neurosurg 43:523-534, 1975

9. Marmarou A, Shulman K, Rosende RM: A nonlinear analysis of the cerebrospinal fluid system and intracranial pressure dynamics. J Neurosurg 48:332-344, 1978

10. Martin AJ, Drake JM, Lemaire C, Henkelman RM: Cerebrospinal fluid shunts: flow measurements with MR imaging. Radiology 173:243-247, 1989

11. O’Brien DF, Taylor M, Park TS, Ojemann JG: A critical analysis of 'normal' radionucleotide shuntograms in patients subsequently requiring surgery. Childs Nerv Syst 19:337-341, 2003

12. Ohara S, Nagai H, Matsumoto T, Banno T: MR imaging of CSF pulsatory flow and its relation to intracranial pressure. J Neurosurg 69:675-682, 1988

13. Patwardhan RV, Nanda A: Implanted ventricular shunts in the United States: the billion-dollar-a-year cost of 
hydrocephalus treatment. Neurosurgery 56:139-145, 2005

14. Rice HE, Frush DP, Farmer D, Waldhausen JH: Review of radiation risks from computed tomography: essentials for the pediatric surgeon. J Pediatr Surg 42:603-607, 2007

15. Simon TD, Riva-Cambrin J, Srivastava R, Bratton SL, Dean JM, Kestle JRW: Hospital care for children with hydrocephalus in the United States: utilization, charges, comorbidities, and deaths. J Neurosurg Pediatr 1:131-137, 2008

16. Stein SC, Guo W: Have we made progress in preventing shunt failure? A critical analysis. J Neurosurg Pediatr 1:40-47, 2008

17. Tuli S, Drake J, Lawless J, Wigg M, Lamberti-Pasculli M Risk factors for repeated cerebrospinal shunt failures in pediatric patients with hydrocephalus. J Neurosurg 92:3138, 2000

18. Vassilyadi M, Tataryn ZL, Matzinger MA, Briggs V, Ventureyra ECG: Radioisotope shuntograms at the Children's Hospital of Eastern Ontario. Childs Nerv Syst 22:43-49, 2006

19. Wong JM, Ziewacz JE, Ho AL, Panchmatia JR, Bader AM, Garton HJ, et al: Patterns in neurosurgical adverse events: cerebrospinal fluid shunt surgery. Neurosurg Focus 33(5):E13, 2012

\section{Disclosures}

Mr. Pennell is an employee of Transonic Systems Inc.

\section{Author Contributions}

Conception and design: Krishnamurthy, Pennell, Kaufman. Acquisition of data: all authors. Analysis and interpretation of data: Krishnamurthy, Pennell. Drafting the article: Krishnamurthy, Pennell, Yi. Critically revising the article: Krishnamurthy, Yi, Kaufman. Reviewed submitted version of manuscript: Krishnamurthy, Pennell, Yi. Approved the final version of the manuscript on behalf of all authors: Krishnamurthy. Administrative/ technical/material support: Pennell, Kaufman. Study supervision: Krishnamurthy, Kaufman.

\section{Correspondence}

Satish Krishnamurthy, Department of Neurosurgery, SUNY

Upstate Medical University, Jacobsen Hall, 6th Fl., 175 Elizabeth Blackwell St., Syracuse, NY 13210. email: krishnsa@upstate.edu. 